\title{
On flow and work hardening expression correlations in metallic single crystal plasticity
}

\author{
P. Franciosi
}

\author{
Laboratoire PMTM CNRS, Université Paris-Nord, Avenue J.B. Clément, \\ 93430 Villetaneuse France \\ (Reçu le 27 mai 1987, révisé le 29 octobre 1987, accepté le 2 novembre 1987)
}

Résumé. - De la comparaison des principaux modèles phénoménologiques décrivant 1 'écoulement plastique (loi de Schmid, approche dépendante de la vitesse, modèle de percolation) et 1'écrouissage (loi d'écrouissage de déformation, loi généralisée) des monocristaux métalliques, on propose un cadre général duquel les associations usuelles loi d'écoulement-loi d'écrouissage utilisées, peuvent $s^{\prime}$ interprêter comme des cas particuliers associés à des hypothèses que $l^{\prime}$ on $s^{\prime} e f f o r c e$ d'établir.

Pour justifier cette formulation générale, on propose une description microstructurale des mécanismes mis en jeu, basée sur l'introduction dans l'expression de la densité des dislocation primaires et celle du glissement sur les différents systèmes, d'une distribution, sur chacun de ces systèmes, de segments de dislocations activables.

I1 en ressort notamment que dans le cadre de la loi de Schmid, la loi d'écrouissage de déformation est le cas limite de cette description correspondant à une distribution de Dirac pour les segments de dislocations - leur loi de déplacement obeissant également à une fonction de ce type - alors que pour toute autre distribution, une loi d'écrouissage généralisée apparait plus satisfaisante. Une telle loi d'écrouissage généralisée, où les vitesses de glissement ne sont plus les seuls paramètres d'écroulssage, apparait nécessaire pour prendre en compte la contribution à l'écrouissage des systèmes inactifs, ce qui justifie des travaux antérieurs réalisés dans ce but. Une dépendance en vitesse de l'écoulement s'exprime ici par une loi de déplacement pour les segments de dislocations qui n'est plus de type Dirac. Si le seuil d'écoulement plastique n'est supposé qu'asymptotiquement atteint, on retrouve une forme connue de représentation dépendante de la vitesse.

Abstract. - From a comparison of the main phenomenological models describing the single crystal plastic flow (Schmid law, rate dependent approach, percolation model) and work hardening (strain hardening law,generalized hardening), one proposes a general frame of which the usual plastic flow-hardening law associations can be interpreted as particular cases associated with assumptions one tries to specify.

To justify this general formulation, one proposes a microstructural description of the involved me sions on the different systems, of a dislocation segment distribution on each slip system to be activated.

It mainly comes out that, within the Schmid law frame, the strain hardening law is the limit case of this representation, corresponding with a Dirac distribution for the dislocation segments - the displacement law of which also obeying to such a Dirac function - while for any other distribution, a generalized hardening law is more convenient. Such a generalized hardening law, where the slip rates are no more the only hardening parameters, appears necessary to account for the hardening contribution of the inactive systems, what justifies previous works performed with this aim. A rate dependency of the flow is here expressed by a segment displacement law which is no more a Dirac like function. If the plastic flow threshold is assumed only asymptotically reached, one finds again a known type of rate dependent representation.

\section{INTRODUCTION.}

The descriptions of the elementary plastic flow and hardening processes at the microstructural scale are still characterized by more or less question- nable approximations which are difficult to avoid because of the complexity of the real behaviour.

At the appropriate macroscopic level of the crystallographic mechanisms for single crystal plasti- 
city, several phenomenological descriptions of flow and work hardening have been developped, each one being justified with the help of a simple microstructural behaviour skeme.

Basically, the major phenomenological descriptions developped so far are the Schmid law and the rate dependent approach for flow, and the strain hardening law and a generalized hardening expression for work hardening. Statistical flow descriptions have been also investigated, as a percolation model for instance, although not as much used so far.

From a comparison of these different phenomenological approaches, one proposes in part I a general representation for both the plastic flow and the work hardening conditions, of which the usual phenomenological models mentionned above can be interpreted as particular cases. The part II presents a microstructural background to support this generalized plastic flow description, based on the introduction, in both the slip and the primary dislocation density expressions, of a dislocation segment distribution per unit volume and on each slip system. In part III, the various approximations leading to the usual models reported in part $I$ are discussed with regard to this description.

I. FUNDAMENTAL BACKGROUND AND USUAL FLOW DESCRIPTIONS.

In pure metals, plasticity is essentially related with dislocation behaviour, that is dislocation creation, multiplication, motion and interactions. If both slip and work hardening involve these various mechanisms, they differently depend on them, and this is the source of one major difficulty in single crystal plasticity modelling.

Typically, we can assume that on any $g$ system, the dislocations are of two kinds : those emitted from active sources under stress and those resulting from interactions between these emitted loops and any encountered dislocation. The former will be called primary dislocations and the latter, the secondary dislocations or the interaction products.

If plastic flow is basically a function of the primary dislocation density, work hardening depends on an obstacle density, the composition of which includes both primary and secondary density elements through a complex relation. However, at this dislocation density scale, the work hardening expression is the question which receives the more satisfying answer from experimental data and we begin with it.

\section{I.1. Work hardening and obstacle density}

Comparing with the case of a dislocation motion through point defects of various strengths, the critical resolved shear stress (CRSS) to move a dislocation through a forest density has been of ten written (Zarka 1972, Lavrentev 1976):

$$
\tau_{c}^{g}=\mu b \sum_{\ell=1}^{N} \alpha_{\rho}^{g l_{\rho} \ell(n / 2)} \underbrace{1 / n}_{0} \quad 1<n<2
$$

where $\rho_{0}^{l}$ is the obstacle density of the $\ell$ system (say dislocations of $a b^{l}$ Burgers vector) and $\alpha$ a dislocation interaction coefficient matrix. For $n=2$, the incremental related form is:

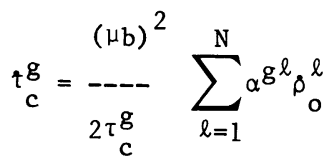

generalizing the Taylor isotropic first law (1934).

Typically, the obstacle density belonging to a $g$ system, can be dissociated into two main contributions as schematized on Fig. 1:

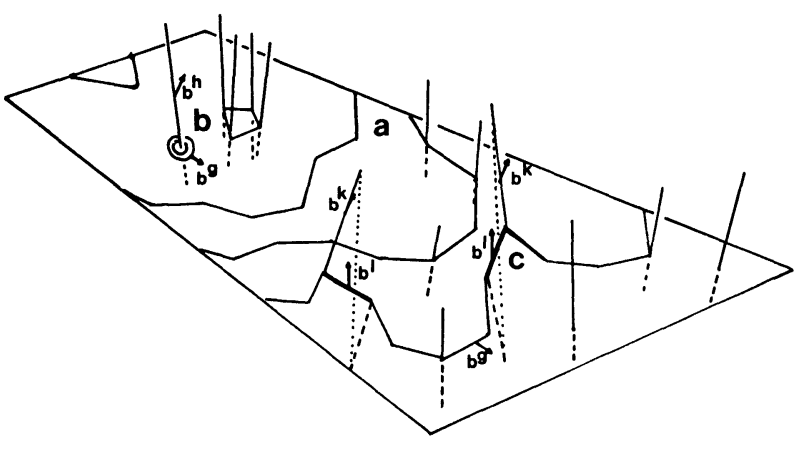

a) primary mobile loops

b) secondary loops, c) junctions

Fig. 1. The obstacle density components.

- the primary still mobile loops $\rho_{\mathrm{m}}^{\mathrm{g}}$ on $\mathrm{g}$ : the mobile dislocation density $\rho_{m}$ differs from the primary density $\rho$ created from the onset of loading, by a rearranged density $\rho_{*}=\rho-\rho_{m}$, the variation rate of which being assumed equal to the primary density one, under the assumption of steady state $\rho_{m}=0$.

The rearranged density is the one involved in the interaction processes.

- the interaction products of the primary loops with dislocations of the other systems, mainly with the dislocations piercing the slip planes of the considered $g$ system: the primary mobile loops can let a 
secondary loop of same Burgers vector around the tree obstacles, and continue to move on. This is the interaction process usually called for to express the rearranged density $\rho_{*}$, the variation rate of which being assumed proportionnal to $\rho_{\mathrm{m}}$.

A general expression for these secondary loops can be written as:

$\rho_{L}^{g}=\sum_{h \neq g} C^{g g h}\left(\left(\rho_{0}+\rho_{m}^{g}\right)\left(\rho_{0}+\rho^{h}\right)-\rho_{o}\right)$

into which $\rho_{0}$, without upper index, is the initial dislocation density on each $g$ system, assumed identical on all systems in the absence of all prestrain history: the mobile loops can also interact with the encountered tree so as to make junction products, which more unlikely allow further motion. (Anihilation events contribute to hardening in reducing the interacting primary density by a fraction which is here assumed constant and not considered in the following). This interaction type can be considered as a gross representation of a substructure hardening contribution. A general expression for it can be given under the form:

$\rho_{J}^{g} \sum_{h \neq k \neq g} \sum_{C^{g h k}}\left(\left(\rho_{0}+\rho_{*}^{h}\right)\left(\rho_{0}+\rho^{k}\right)-\rho_{0}\right)$

with a double summation over the slip systems of the structure.

Primary loops can also interact with secondary dislocations, to create second order interaction products and so on. This has been discussed in details previously (see Franciosi 1985). Then, at

this first interaction order, the obstacle density expression of $b^{l}$ Burgers vector is:

$$
\rho_{\mathrm{o}}^{\ell}=\rho_{\mathrm{m}}^{\ell}+\rho_{\mathrm{L}}^{\ell}+\rho_{\mathrm{J}}^{\ell}
$$

Involving the two components $\rho_{m}^{\ell}$ and $\rho_{*}^{\ell}$ of the primary dislocation densities on each $\ell$ system.

Now, although the exact primary dislocation density expression is ignored, it must at least be related with slip.

Basically the slip rate is either assumed to be a function of the primary dislocation density times an average dislocation velocity V, (Orowan 1940):

$$
\dot{\gamma}=\rho b V
$$

or alternatively (when the creation of dislocations is a process much slower than their propagation), a function of the primary dislocation production rate time an average displacement law D:

$$
\dot{\gamma}=\dot{\rho} \mathrm{bD}
$$

In (6), the involved density is generally assumed to be the mobile one $\left(\rho=\rho_{\mathrm{m}}\right)$. Then the steady state assumption $\rho_{\mathrm{m}}=0$ allows to write the obstacle density rate $\dot{\rho}_{0}$, when identified with $\dot{\rho}_{*}$, as:

$$
\dot{\rho}_{\mathrm{o}}=\dot{\rho}_{\star}=\rho_{\mathrm{m}} \mathrm{V} / \mathrm{D}=\dot{\gamma} / \mathrm{bD}
$$

introducing now $D$ as an average finite free path between interactions. In (7) one has $\dot{\rho}=\dot{\rho}_{m}+\dot{\rho}_{*}$, say the primary dislocation density (created from $t=0$ ) variation rate, and not only the variation rate of the mobile ones. For steady state $\dot{\rho}_{\mathrm{m}}=0$, and still identifying the obstacle density rate $\dot{\rho}_{0}$ to $\dot{\rho}_{*}$, one directly obtains:

$$
\dot{\rho}_{0}=\dot{\rho}_{\star}=\dot{\gamma} / \mathrm{bD}
$$

with $D$ the average dislocation displacement law.

These simple derivations of a $\dot{\rho}_{0}(\dot{\gamma})$ relation, which look similar but are different, remain very questionable. However, both these descriptions support the phenomenological strain hardening law:

$$
\dot{\tau}_{c}^{g}=\sum_{\ell=1}^{N} h^{g l} \dot{\gamma}^{\ell}
$$

where hardening is represented by a hardening moduli matrix $h$ with one, two or more differents terms according to various anisotropy assumptions.

If on the contrary the obstacle density rates are not assumed to only depend on the slip rates $\dot{\gamma}^{l}$, but also on extra parameters say $\dot{z}^{l}$ (not specified now), the strain hardening law has to be generalized into:

$$
\dot{\tau}_{c}^{g}=\sum_{\ell=1}^{N}\left(h^{g l} \dot{\gamma}^{l}+k^{g l} \dot{z}^{l}\right)
$$

Such a generalized hardening law, introduced previously (Franciosi 1985,1987) for a special $\dot{z}^{l}$ choice, is considered later on in a more general way.

If the work hardening law can be generalized as (11), the $h$ (or $h$ and $k$ ) moduli identification is a separate difficulty since they in general diffe- 
rently depend on the strain history. Their description depends on the relation between the primary density and the hardening parameters $\left(\dot{\gamma}^{l}\right.$, or $\dot{\gamma}^{l}$ and $\dot{z}^{l}$ ), and for instance from (2) and with (8) or (9), they would take the form:

$$
\mathrm{h}^{\mathrm{gl}}=\frac{(\mu \mathrm{b})^{2}{ }_{\alpha} \mathrm{gl}}{2 \tau_{\mathrm{c}}^{\mathrm{g}} \mathrm{bD}^{\ell}}
$$

with a different $D^{\ell}$ value for each $\ell$ system.

We now summarize the usual plastic flow phenomenological expressions derived so far, to point out the common features and the differences.

\section{I.2. Usual plastic flow descriptions}

We first recall the percolation model developped by Kocks $(1966,1967)$, which is more physical than phenomenological, and then the major descriptions i-e the rate dependant approach and the Schmid law.

- a. The percolation model

As developped by Kocks, for a dislocation of a $g$ system moving through a two dimensionnal array of point obstacles, like in Fig. 2, a calculation of the fraction of penetrable obstacles as a function

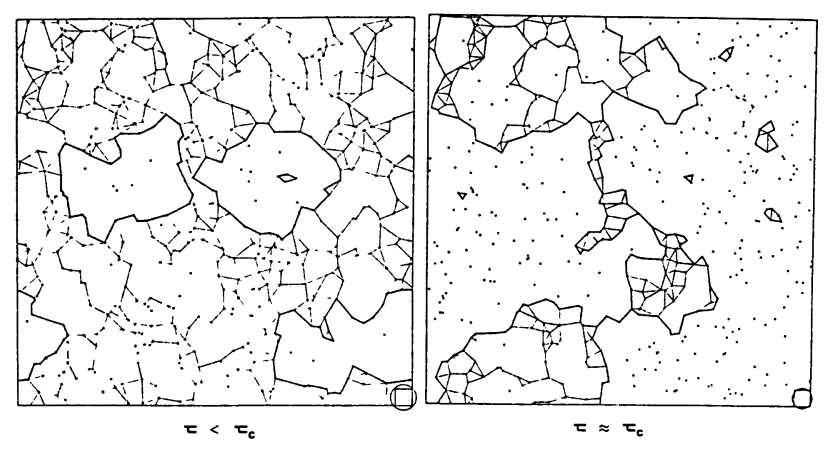

Fig. 2. Dislocation motion through point obstacles

of the ratio $\zeta^{g}=\tau^{g} / \tau_{c}^{g}$ has given the relation:

$$
\mathrm{F}(\zeta)=\exp ^{-\left(\zeta^{-n}\right)} \quad \mathrm{n}=1,2,3
$$

which defines a percolation function $F(\zeta)$, from which the slip increment due to a d $\zeta$ increment can be expressed under the form of the geometrical series:

$\mathrm{d} \gamma=\mathrm{bdA}(\mathrm{F}) / \mathrm{V}=\mathrm{baNdF}(1+\mathrm{cF}(1+\ldots=\mathrm{baNdF} /(1-\mathrm{cF})$
In (14), a is the average cell area, $N$ the segment number per untt volume and $c+1$ the cell side number. In this statistical displacement description, and when the loop motion is assumed infinitely free, slip can become infinite for $F=1 / c$, say when one at least of the $c$ sides of the cells obtained in joining the neighbouring point obstacles is penetrable (the calculated $c$ value for a random array of obstacles is between 2.5 and 3.5). The area swept is given by $a /(1-c F)$ and therefore $N d F$ is the active segment number at $\zeta$. Then, the $\mathrm{dF}(\zeta)$ increment in (14) can be Identified with the segment fraction activable during $d \zeta$. As detailed in Kocks (1967), in the slip increment expression written under the form:

$$
d \gamma=b_{o}(N d F+F d N)
$$

$\mathrm{dN}$ is the number per unit volume of new occupied links between obstacles since $F$ was raised, and reduces to (14) when this increase is assumed strictly dependant on the slip increment so that:

$$
\mathrm{dN}=\mathrm{cd} \gamma / \mathrm{a}_{\mathrm{o}} \mathrm{b}
$$

When $\zeta$ is not monotonically increasing during the load process, this description has to be completed, by assumptions on the backward loop motion. Steady state is here reached at $F=F_{c}$, and if then $\zeta$ is only increased to ensure this equality, the flow in steady state is described by the condition $\mathrm{d} \zeta=0$, $d_{\tau}=d \tau_{c}$. A question, discussed later on is whether the critical $F_{c}$ value is reached or is only an asymptotical limit, when hardening is considered.

- b. The rate dependent approach

This approach, summarized for instance in Asaro and Needleman (1983), is supported by the assumption that the slip rates on all the easy glide systems of the structure are given by the Orowan law (6), with the dislocation velocity taken under the form :

$$
v^{g}=v_{o}\left(\tau^{g} / \tau_{c}^{g}\right)^{m}
$$

derived from experimental measurements giving a linear relation between $v$ and $\tau^{m}$, assuming in addition a maximum velocity which would be related with the sound velocity. This velocity expression accounts for the two major experimental features 
which are the shear stress dependency form and the upper limit, and the slip rate is therefore written:

$$
\dot{\gamma}^{g}=\dot{\gamma}_{o}\left(\tau^{g} / \tau_{c}^{g}\right)^{m}
$$

using a power law in $\zeta$ as suggested by Hutchinson (1976), with $\dot{\gamma}_{0}$ a constant reference slip rate.

As in the percolation model, the ratio $\zeta^{g}=\tau^{g} / \tau_{c}^{g}$ appears to be a fundamental parameter in the flow description, although introduced in a different way.

However, since such a rate dependent description involves here a real time derivative and not a kinematical one, the derivation of a general frame for the single crystal plastic behaviour description will not be able to include it formally.

However, when $\dot{\gamma}_{0}$ is no more chosen constant but depends on a flow parameter, the approach is no more rate dependent, what will serve later on.

In this rate dependent description, the slip rate asymptotically tends towards the reference value, and the steady state is never reached. The use of the Orowan law under the form (6) can therefore appear debatable.

- c. The Schmid law

This representation, which is the oldest one (Schmid and Siebel 1931) but still the most widely used, assumes that slip on a $\mathrm{g}$ system is allowed only once the applied resolved shear stress on it reaches a critical value $\tau_{c}^{g}$, and continues if the applied shear stress rate remains equal to the critical resolved shear stress rate. One can write:

$$
\begin{aligned}
& \mathrm{d} \gamma^{\mathrm{g}} \geqq 0 \quad \text { if } \quad \tau^{\mathrm{g}}=\tau_{c}^{\mathrm{g}} \quad \text { and } \mathrm{d} \tau^{\mathrm{g}}=\mathrm{d} \tau_{c}^{\mathrm{g}} \\
& \mathrm{d} \gamma^{\mathrm{g}}=0 \quad \text { if not, say }\left\{\begin{array}{c}
\tau^{\mathrm{g}}<\tau_{c}^{g} \\
\text { or } \tau^{g}=\tau_{c}^{g} \text { and } \mathrm{d} \tau^{\mathrm{g}}<\mathrm{d} \tau_{c}^{g}
\end{array}\right.
\end{aligned}
$$

Since this criterion involves only $\tau^{g}$ and $\tau_{c}^{g}$, one can rewrite it, in using the $\zeta^{g}$ ratio appeared in the previous descriptions, under the form:

$$
\begin{aligned}
& \mathrm{d} \gamma^{\mathrm{g}} \geqq 0 \quad \text { if } \zeta^{\mathrm{g}=1} \text { and } \mathrm{d} \zeta^{\mathrm{g}}=0 \\
& \mathrm{~d} \gamma^{\mathrm{g}=0} \quad \text { if not, say }\left\{\begin{array}{c}
\zeta^{\mathrm{g}}<1 \\
\text { or } \begin{array}{r}
\zeta^{\mathrm{g}}=1 \text { and } \mathrm{d} \zeta^{\mathrm{g}}<0
\end{array}
\end{array}\right.
\end{aligned}
$$

This criterion, introduces the concept of yield surface below which no microstructural events are accounted for, and defines a macroscopic yielding, before which both slip and work hardening are neglected. In opposition with the two previous approaches, this criterion neglects the transition domain, and consideres only the steady state plasticity.

From these plastic flow descriptions, some general features can be now pointed out.

\section{I.3. Common features of the flow descriptions}

The essential point is that in all these descriptions, the same undimensional parameter $\zeta^{g}=\tau^{g} / \tau_{c}^{g}$ appears predominant in the slip expression, the variation of which with $\zeta^{g}$ has always the same sharply increasing form obeying (as shown on Fig.3), to the relation (in the $\{0,1\} \zeta^{g}$ domain):

$$
\mathrm{d} \gamma^{\mathrm{g}}=\frac{\mathrm{bA}_{0} \partial \mathrm{S}\left(\zeta^{\mathrm{g}}\right)}{\mathrm{V} \partial \zeta^{\mathrm{g}}}
$$

For the percolation model one has:

$$
S\left(\zeta^{g}\right)=\ln \left(\left(1-c F\left(\zeta^{g}\right)\right)^{-1}\right)
$$

(identifying the asymptotical 1imit with the $\zeta^{8}=1$ value, say $c=\exp (1)$ ) when $F$ is the function (13);

For the Schmid law one has:

$$
S\left(\zeta^{g}\right)=\Delta\left(\zeta^{g}\right)
$$

with $\Delta\left(\zeta^{g}\right)$ the Dirac function, and, in the description derived from the rate dependent approach with $d \zeta^{g}$ for $\dot{\gamma}_{0} d t$, one has:

$$
\mathrm{S}\left(\zeta^{\mathrm{g}}\right)=\left(\zeta^{\mathrm{g}}\right)^{\mathrm{m}} / \mathrm{m} \quad \mathrm{m}>1
$$

say the power law (18).

In the slip increment expression (21), the load process dependency is assumed reducible to the $\zeta^{g}$ parameter. A more general expression would at least involve also the timelike parameter which defines the load proces, say $\theta$, leading to write:

$$
d \gamma^{g}=\gamma_{, \theta}^{g} d \theta+\gamma_{, \zeta^{g}}^{g} d \zeta^{g}
$$

(with the usual conventions for the partial derivatives), like the expression (15) for instance 


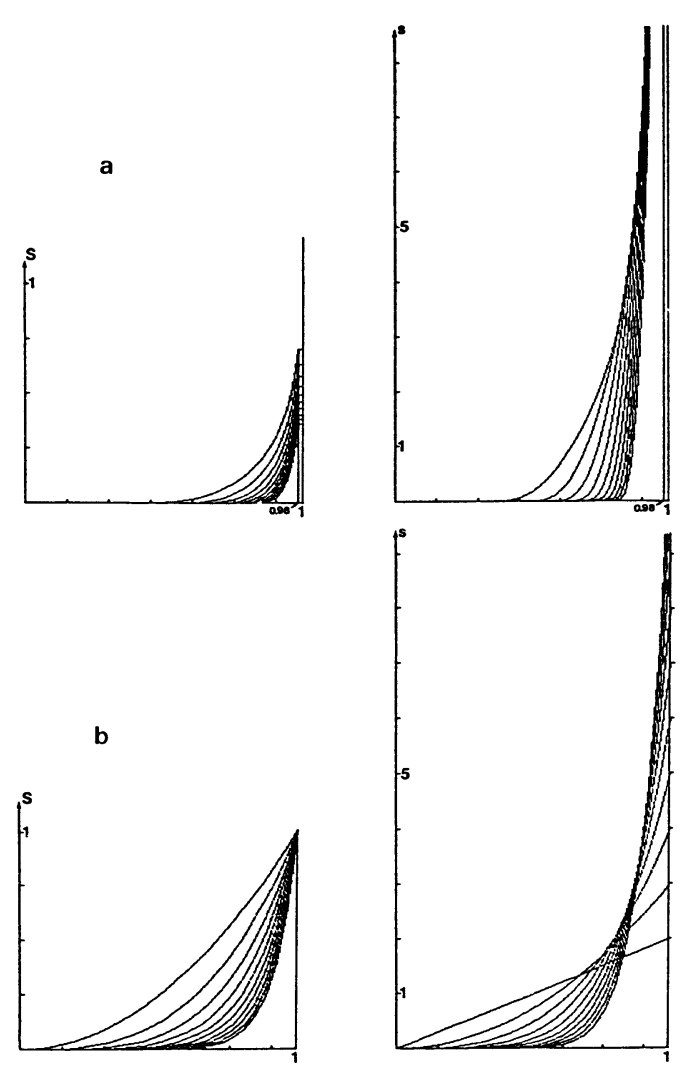

Fig. 3. The $\mathrm{S}(\zeta)$ functions (22) a, (24)b and derivatives.

if the $\mathrm{dN}$ term does not only depend on slip.

Now, when one chooses the microstructural hardening law (2), instead of the strain hardening law (10), one must also express the primary dislocation density evolution during the loading process. From what preceeds, one can expect an expression for $\mathrm{d} \rho^{\mathrm{g}}$ similar to the one in (25) for the slip increment, say:

$$
\mathrm{d} \rho^{\mathrm{g}}=\rho_{, \theta}^{\mathrm{g}} \mathrm{d} \theta+\rho^{\mathrm{g}}, \zeta^{\mathrm{g}} \mathrm{d} \zeta^{\mathrm{g}}
$$

We now investigate the microstructural background allowing to derive such expressions for $d \rho^{g}$ and $d \gamma^{g}$, and the $d \rho^{g}$ versus $d \gamma^{g}$ relation.

\section{MICROSTRUCTURAL ANALYSIS}

To derive a general slip versus primary dislocation density relation, we first introduce the concept of dislocation segment distribution by unit volume and for each slip g system, which will appear in both the slip and the primary dislocation density expressions.

\section{II.1 The dislocation segment distribution}

Defining, as in Kocks (1966), the dislocation segments per unit volume on each $g$ system as the occupied links between near neighbour point obstacles in the slip planes (see Fig. 2), we consider that the primary dislocation segment density can be represented for each $g$ system, by a density function $s\left(z^{g}\right)$, and so that the mobile fraction at $\theta$, takes the form:

$$
S\left(z^{g}\right)=\int_{-z^{g}=0}^{z^{g}} s\left(\bar{z}^{g}\right) d \bar{z}^{-g}
$$

with $z^{g}(\theta)$ a positive undimensionnal parameter specified in what follows: since, within such a definition, the segments can be assumed distributed around any critical length $l_{c}^{g}$, related with the current mean length of these links between obstacles $\left\langle l^{g}\right\rangle$, and which evolves like it with the hardening, one has:

$$
z^{g}=\ell_{c}^{g} / \ell^{g}=\tau^{g} / \tau_{c}^{g}
$$

for segments of length $\ell^{g}$ activated under an applied shear stress $\tau^{g}(\theta)$ according to:

$$
\ell^{g}=\mu \mathrm{b} / 2 \pi \tau^{g} \text { with } \ell_{c}^{g}=\mu b / 2 \pi \tau_{c}^{g}
$$

This leads to the distribution $\mathrm{S}\left(\mathrm{z}^{\mathrm{g}}\right)$, and introduces the $\tau^{g} / \tau_{c}^{g}$ ratio appearing in part II. Then, in (25) and in (26), one has the identity $z^{g}=\zeta^{g}$. For instance, the critical length $\ell_{c}^{g}$ can be chosen as the length of the greatest family of segments, so that at $\ell^{g}=l_{c}^{g}, s(1)=s_{\max }$. One has, in general:

$$
\left\langle\tau^{\mathrm{g}}\right\rangle=(\mu \mathrm{b} / 2 \pi)\left\langle 1 / \ell^{\mathrm{g}}\right\rangle \neq \mu \mathrm{b} / 2 \pi\left\langle l^{\mathrm{g}}\right\rangle
$$

with the relations between critical and mean values:

$$
\left\langle l^{g}\right\rangle=l_{c}^{g}\left\langle 1 / z^{g}\right\rangle \quad\left\langle\tau^{g}\right\rangle=\tau_{c}^{g}\left\langle z^{g}\right\rangle
$$

In (26), any statistically reasonable density function can be chosen for $s\left(z^{g}\right)$, provided that the $z^{g}$ definition domain is specified: if the largest $\ell^{g}$ value, related with the crystal dimension or the grain size, can be chosen infinite compared with the 
$\ell_{c}^{g}$ value, the lowest value can be either chosen to be zero or $l_{c}^{g}$ if most of the segments are assumed activated at this critical value. Therefore the $z^{g}$ validity domain can be elther $\{0,1\}$ or $\{0, \infty\{$. Now, the critical length could have been defined as the length of the smallest segments, instead of the length of the greatest number of segments. Limiting the density to the $\{0,1\}$ domain simply defines the critical stress as the stress at which all the segments are active, what is an upper bound.

Since $z^{g}$ is not necessarily monotonically increasing, the active source fraction described by (26) may increase or decrease with regard to $\theta$, as shown on Fig. 4.

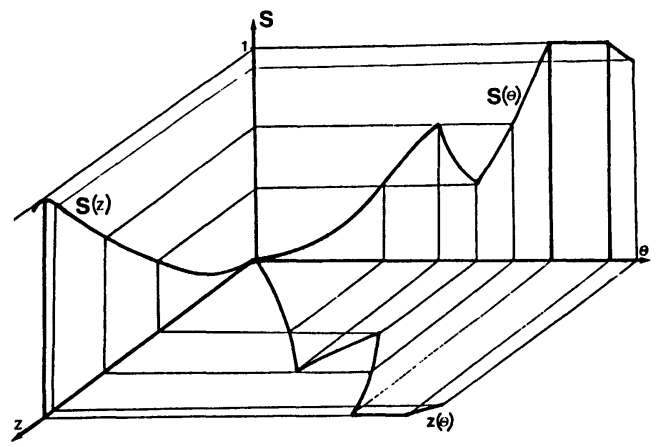

Fig.4. The $S\left(z^{g}\right), S(\theta)$ and $z^{g}(\theta)$ relative variations

Among possible density functions of these kinds, one can mention two exemples (the $g$ index is omitted in what follows):

- the power law shown on Fig.3, defined by:

$\mathrm{s}(\mathrm{z})=\mathrm{pz} \mathrm{p}^{\mathrm{p}-1}$

$S(z)=z^{p}$

$$
\mathrm{p}>1, \quad \mathrm{z} \in\{0,1\}
$$

where the highest segment density corresponds with the shortest ones. The $z$ mean value is $p /(p+1)$ and the $\mathrm{z}^{-1}$ one is $\mathrm{p} / \mathrm{p}-1$, what gives:

$$
\ell_{c}=\langle l\rangle(p-1 / p) \quad \tau_{c}=\langle\tau\rangle(p+1 / p)
$$

- the exponential negative function (Fig. 5a):

$s(z)=\beta q z^{-(\beta q)} \exp ^{-\beta\left(z^{-q}\right)} \quad z \in\{0, \infty\{$

$S(z)=\exp ^{-\beta\left(z^{-q}\right)} \quad, \beta=q+1 / q, q>0$

which becomes, if truncated at $z=1$ (Fig. 5b):

$$
\begin{array}{ll}
s(z)=\beta q z^{-(\beta q)} \exp ^{\beta\left(1-z^{-q}\right)} & z \in\{0,1\} \\
s(z)=\exp ^{\beta\left(1-z^{-q}\right)} \quad, \beta=q+1 / q, & q>0
\end{array}
$$

Here too, mean and critical length values are not necessarily equal.

These density and distribution functions have all the same limit, when $p$ and $q$ respectively is infini-
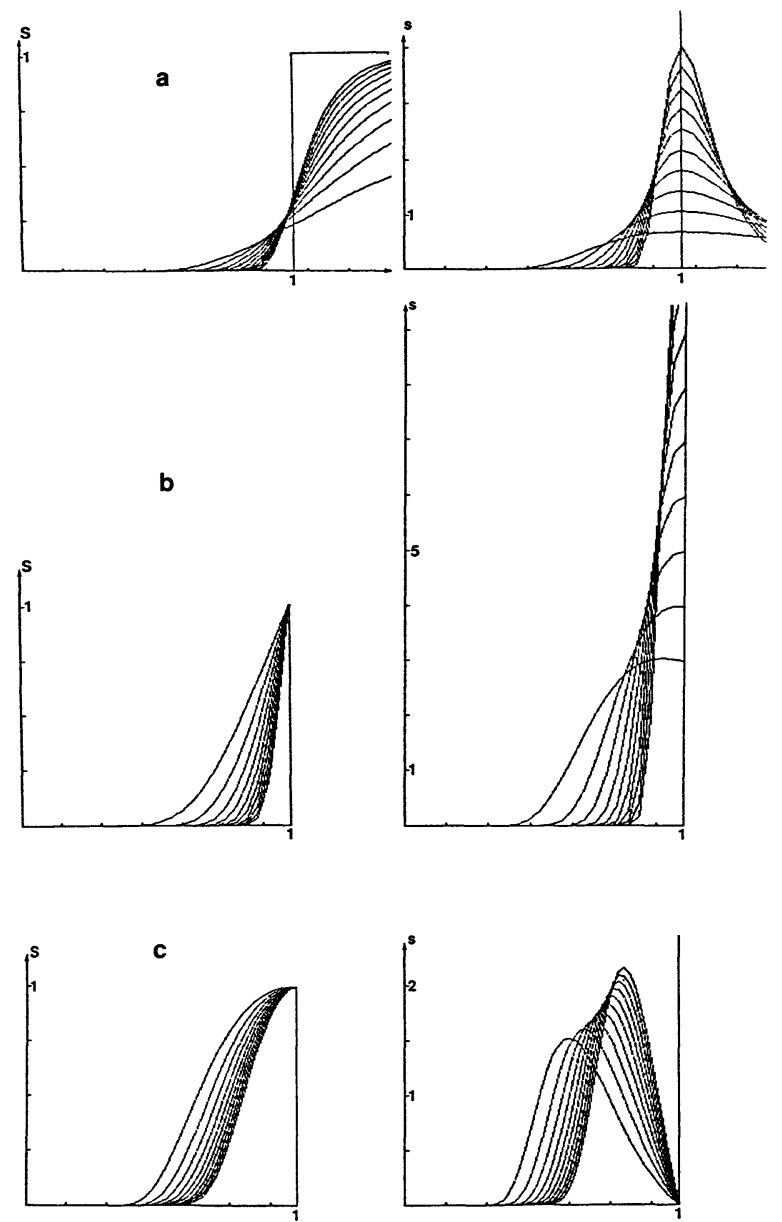

Fig. 5 Distribution functions (33)a, (34)b, (36)c and derivatives

tely high, which is the Dirac peak density:

$$
\begin{array}{llll}
s(z)=0 & \text { and } & S(z)=0 & \text { if } z \neq 1 \\
s(z)=\delta & \text { and } & S(z)=1 & \text { if } z=1
\end{array}
$$

In this singular limit case, $\ell_{c}=\langle\ell\rangle, \tau_{c}=\langle\tau\rangle$ and all the dislocation segments are active under the same shear stress, while no activity is allowed 
as long as this shear stress is not reached.

However, when the distribution is truncated at $z=1$, any function of $z$, monotonically increasing between zero and unity can be used to describe the $S$ distribution, and in particular, the law (Fig.5c):

$$
S(z)=\exp ^{-\beta\left(1-z^{-1}\right)^{2}}
$$

is as relevant as the previous ones. We now express the slip versus primary loop density relation.

II.2. Primary dislocation density and slip

\section{expressions}

Considering any $\mathrm{g}$ system of the concerned crystal structure, still omitting the $g$ index for brevity, if a segment family $s(\bar{z}) d \bar{z}$ activated at $\bar{z}=z(\bar{\theta})$, allows some new segment production (by new source activation) between $\bar{\theta}$ and $\theta$, the increase of mobile segment number per unit volume is:

$$
\operatorname{dn}(\bar{z}, \theta)=\left(\nu_{0}+\int_{\bar{\theta}}^{\theta} v(\theta, \bar{z}) d \theta\right) s(\bar{z}) d \bar{z}
$$

with $v$ the segment production rate and $\nu_{0}$ the initial segment number. The dislocation density increase per unit area is:

$$
\mathrm{d} \rho(\bar{z}, \theta)=\left(\nu_{0}+\int_{\bar{\theta}}^{\theta} \operatorname{vd} \theta\right) l_{s}(\bar{z}) d \bar{z}
$$

with $l=\langle\ell\rangle_{z}$, the mean segment length.

The associated slip increment is then:

$$
\mathrm{d} \gamma(\bar{z}, \theta)=b\left(\nu_{0}+\int_{\bar{\theta}}^{\theta} \operatorname{vd} \theta\right) a s(\bar{z}) d \bar{z}
$$

with $a=\langle a\rangle_{z}$, the swept area by each segment.

Then, for all the active sources at $\theta$, and first when the unknown function $z(\theta)$ is monotonically increasing, the total primary dislocation density and the total slip are given by, (with $\theta *=\theta-\bar{\theta})$ :

$$
\rho(\theta, z)=\int_{\bar{z}=0}^{z(\theta)}\left\langle L_{c}\right\rangle_{\theta *} s(\bar{z}) d \bar{z}=\left\langle\left\langle L_{c}\right\rangle_{\theta *}\right\rangle_{z} S(z)
$$

and:

$$
\gamma(\theta, z)=b \int_{\bar{z}=0}^{z(\theta)}\left\langle A_{c}\right\rangle_{\theta *} s(\bar{z}) d \bar{z}=b\left\langle\left\langle A_{c}\right\rangle_{\theta *}\right\rangle_{z} S(z)(40 b)
$$

say, of the syntlietic form:

$$
\rho=\left\langle L_{c}(\theta, z)\right\rangle S(z)=L S
$$

$$
\gamma=b<A_{c}(\theta, z)>S(z)=b A S
$$

with $\mathrm{D}=\mathrm{A} / \mathrm{L}$ the mean primary dislocation displacement law, generally depending on $z$ and $\theta$.

A similar analysis of slip and primary dislocation density has been recently reported in Marzik et al (1987) from an experimental investigation of the dislocation segment distributions.

The incremental form derived from (40) is then:

$$
\begin{aligned}
& d \rho=L, \theta S d \theta+(L s+L, z S) d z \\
& \mathrm{~d}_{\gamma}=\mathrm{b}\left(\mathrm{A}, \theta^{\mathrm{Sd} \theta}+\left(\mathrm{As}+\mathrm{A}, z^{S) \mathrm{d} z}\right)\right.
\end{aligned}
$$

remaining valid even when $z(\theta)$ is not monotonically increasing. The expressions (43) are a particular case of (25), from which one can write (for $\left.A, \theta^{\neq}\right)$:

$$
\begin{gathered}
\mathrm{d} \gamma \quad\left(A s+A, z^{S}\right) d z \\
{ }^{b}\left(A_{,} S\right) \quad{ }^{A}, \theta^{S}
\end{gathered}
$$

what gives the general slip versus primary dislocation density relation:

$$
\mathrm{d} \rho=\mathrm{d} \mathrm{bA}_{, \theta}^{\mathrm{L}, \theta}+\mathrm{dz}\left(\mathrm{L}, \mathrm{z}^{\mathrm{S}+\mathrm{Ls}-}-\mathrm{L}, \theta^{\left(\mathrm{A}, z^{+A s}\right)}\right.
$$

and with $s^{\prime}=(\mathrm{LS})_{, z}, s^{\prime \prime}=(\mathrm{AS})_{, z}$ :

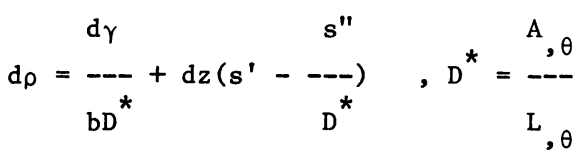

In (45), the dz term only vanishes when:

- $S$ is constant and neither $L$ nor $D$ depend on $z$

- D is constant

The usual strain hardening law (10) is formally valid only for these two particular cases, when $\mathrm{dz}$ is not assumed enough to describe the load process, say when the d $\theta$ dependency does not vanish in (25) and (26).

We now discuss the general flow solution and the allowed approximations in comparison with the usual representations. 


\section{A GENERAL FLOW EXPRESSION AND DISCUSSION}

\section{III.1. A general flow solution.}

Expressing the $\dot{i}^{l}$ parameters in terms of $\dot{\tau}_{c}^{l}$ and $\dot{\tau}^{l}$ from (2), the slip rates being now on defined from the kinematical time $\theta$ so that $\dot{\gamma}=d \gamma / d \theta$, one has:

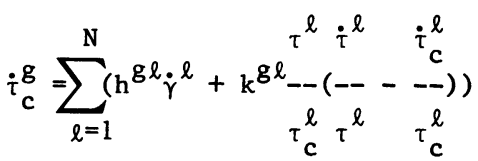

which are the $\mathrm{N}$ hardening conditions allowing to solve the flow problem in addition to the flow conditions (25b), provided a consistent choice in both for the dislocation segment distribution.

As shown previously, (Franciosi 1984), the applied resolved shear stress rate can be written as:

$$
\dot{\tau}^{g}=\sum_{\ell=1}^{N} \Delta h^{g l} \dot{\gamma}^{l}+c^{g}
$$

in small or large strain formalism, where $\Delta \mathrm{h}^{\mathrm{gl}}$ and $\mathrm{C}^{\mathrm{g}}$ are known terms, due to the local conditions in prescribed stress and strain rates (for uniform fields). Once the $\dot{\tau}^{l}$ are eliminated in (46), the hardening law becomes :

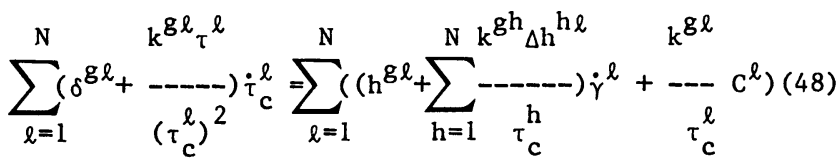
and the left hand side being generally inversible :

$$
\dot{\tau}_{c}^{g}=\sum_{\ell=1}^{N} h^{*} g l \dot{\gamma}^{\ell}+c^{*} g
$$

Finally introducing (47) and (49) in the flow conditions (43b) and after some rearrangements, this gives the system (with $S^{g}, s^{1 g}, s^{\prime g}$ for $S\left(z^{g}\right), s^{\prime}\left(z^{g}\right)$, $s^{\prime \prime}\left(z^{g}\right)$ respectively):

$$
\begin{aligned}
& \Delta \mathrm{h}^{\mathrm{gl}} \quad \mathrm{h}^{{ }^{*} \mathrm{gl}} \mathrm{\tau}^{\mathrm{g}} \quad \mathrm{C}^{\mathrm{g}} \quad \mathrm{c}^{*} \mathrm{~g}_{\tau} \mathrm{g}
\end{aligned}
$$

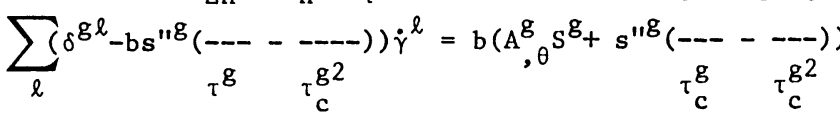

which is the general flow solution in this analysis.

If one calculates first the $\dot{\mathrm{z}}^{l}$ parameters, this must be done using (47) and with (2) written assuming, from (5), $\dot{\rho}_{0}^{l}\left(\dot{\rho}^{l}, \dot{\rho}_{m}^{l}\right) \simeq t^{l h} \dot{\rho}^{h}-$ under the form:

$$
\dot{\tau}_{c}^{g}=\sum_{\ell=1}^{N} T^{g l \cdot \ell}
$$

which gives:

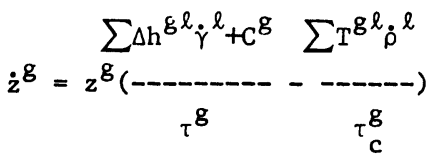

and the system (with $s^{\ell}, s^{\prime l}, s^{\prime \prime}$ for $S\left(z^{\ell}\right), s^{\prime}\left(z^{l}\right)$, $s^{\prime \prime}\left(z^{l}\right)$ respectively):

$$
\begin{aligned}
& \sum_{\ell=1}^{N} \dot{z}^{\ell}\left(\tau_{c}^{g} \delta^{g l}-\left(b \Delta h^{g l} s^{\prime \prime}{ }_{-z} g_{T}^{g l} s^{\prime l}\right)\right.
\end{aligned}
$$

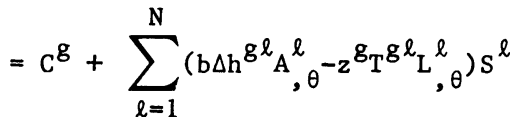

We now discuss the usual phenomenological flow approaches and the convenient work hardening expression with regard to this general description.

III.2. Discussion.

We first consider the Schmid law criterion as the limit case on the flow solution (50).

- a. The Schmid law

When the $\mathrm{S}$ distribution is the Dirac distribution, one has a singular limit case for the flow solution (50): from (43), the slip increment is, for any $g$, zero when $z^{g}<1$, but is undetermined for the potential active systems, $\left(z^{g}=1, \dot{z}^{g}=0\right)$, and is negative infinite for the potential inactive ones $\left(z^{g}=1, \dot{z}^{g}<0\right)$, returning to zero in an instantaneously oscillating mode.

The system (50) leads to:

- for $z^{g}<1, q^{g}=0$

since $S=s=0$ and:

$$
\text { - for } z^{g}=1 \quad, \sum_{\ell=1}^{N}\left(h^{g l}-\Delta h^{g l}\right) \dot{\gamma}^{\ell}=c^{g}
$$

since for $s$ infinite, $h *=h$ and $C^{*}=0$, which are exactly the conditions $\dot{z}^{g}=0,1-e$ the Schmid flow conditions, but for the potential systems being all active. When $z^{g}=1$, the general solution (50) must be completed as the Schmid conditions by unequalities, necessary to allow negative values for $\dot{z}^{\mathrm{g}}$.

In this case the work hardening can be represented by the strain hardening law (10), and the presteady state is entirely neglected. In order to have a rate independant steady state domain, the dislocation 
displacement law D must also be a Dirac like function.

When $s^{\prime \prime}=\mathrm{dS} " / \mathrm{dz}$ in (50) corresponds with a $\mathrm{S}^{\prime}$ Dirac distribution, but not $S$, meaning that the dislocation displacment law D obeys to such a Dirac function (the rate independent limit assumption for the dislocation motion) one has, assuming for simplicity that $D$ only depends on $z^{g}$, for $z^{g}<1$ :

$$
\begin{aligned}
& \dot{p}^{\mathrm{g}}=\mathrm{b}\left(\mathrm{L}_{, \theta}^{\mathrm{g}} \mathrm{D}^{\mathrm{g}} \mathrm{S}^{\mathrm{g}}+(\mathrm{AS}), \mathrm{z}^{\mathrm{z}}\right)=0 \\
& \beta^{\mathrm{g}}={ }^{\mathrm{g}},{ }_{\theta} \mathrm{S}^{\mathrm{g}}+(\mathrm{LS}), \mathrm{z}^{\dot{\mathrm{z}}^{\mathrm{g}}} \neq 0
\end{aligned}
$$

The work hardening law does not reduce here to the strain hardening law (10) but to the generalized form (11).

This description allows to describe the presteady state domain on each slip system, and then the hardening contribution of the inactive systems during plastic straining, what is not only a small strain feature since most of the slip systems remain inactive all along the deformation process. (When $z^{g}$ $=1$, the usual steady state flow description remains valid). A description like (54), but with $\dot{i}^{\mathrm{g}}$ replacing $\dot{z}^{g}$ has been satisfactorily used in previous behaviour simulations of the single crystal response to plastic straining (see Franciosi 1984, 1985, 1987).

We now consider the rate dependent approach to discuss the dislocation segment velocity influence on plastic flow.

- b. The rate dependent approach

We here consider the basic expression (18) into which the reference slip rate $\dot{\gamma}_{0}$ is not a constant but depends either on $\theta$ or $\dot{z}^{g}$ still assuming for simplicity that $D$ in $A$ only depends on $z^{g}$.

For a time like $(\theta)$ dependency, the second term in (43b) vanishes for $s^{\prime \prime}=(A S), z=0$, say when $S^{\prime \prime}$ is again a Dirac function. But now, if $D$ is not a Dirac function - in order to express a dislocation velocity dependency of plastic flow - S must be a Dirac function, and we are again in the Schmid limit description.

For a $\dot{\mathrm{z}}^{\mathrm{g}}$ dependency, the first term in (43b) is zero when $\mathrm{A}_{, \theta}=0$ say when $\mathrm{L}_{, \theta}=0$. Then, $\dot{\rho}^{\mathrm{g}}$ is also only depending on $\dot{z}^{g}$ and one can write:

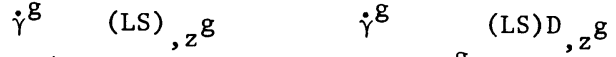

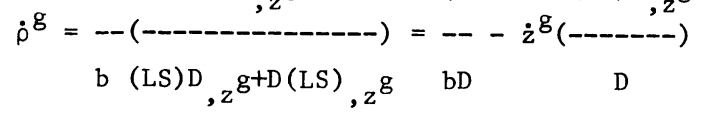

The strain hardening law form (10) can be used here if an extra assumption is given for the $z^{g}$ dependancy of $\mathrm{L}$, what is not necessary a priori in using the generalized hardening form (11), involving $\dot{z}^{g}$. Both work hardening expressions $(10,11)$ can be used, but the strict expression of $\dot{\rho}^{g}$ as a function of the displacement $D$ is only obtained, when $D$ is not constant, if $\dot{z}^{g}$ leads towards 0 . Anyway, even using the strain hardening law (10), the solution requires the calculation of the $\dot{z}^{g}$ terms from (52).

Note that when $D$ is constant, the common $z^{g}$ dependancy of the slip rate and of the primary dislocation density rate on $g$ is due to the segment activation rate $S$, represented by the power law (31), and not to the dislocation segment velocity. The present plastic flow representation is consistent with the rate dependent approach as long as the yield threshold remains an asymptotical limit. With regard to the assumption of such an asymptotical yleld threshold, we now discuss the percolation model and the possibilities of infinite or finite displacements for the dislocation segments.

- c. The percolation model

In the phenomenological representations discussed above, the derived expressions for the $\dot{\rho}^{\mathrm{g}}$ versus $\dot{\gamma}^{\mathrm{g}}$ relation Involve a loop displacement $D$ which must remain finite: On the contrary, the percolation model points out the possibility of an infinite loop displacement on a $g$ system, once on it, a critical value for $z^{g}$ is reached.

Finite displacements for the loops are likely, although the argument of strong back stresses assoclated with expected pile ups, since the experimentally observed built up substructure must be related with the accumulation of weakly mobile interaction products once the mobile segments have moved over a finite path. Separately from this debate, there are two possibilities to account for a finite free path in the percolation model: the first one, dicussed in Kocks (1966) and mentionned in part $I$, is to assume that the critical probability of infinite slip is only reached asymptotically. It would mean that in the system (52), all the $z^{l}$ values remain lower than unity, the $\dot{z}^{l}$ rates tending towards zero asymptotically, more or less rapidly.

The second possibility is to consider that the critical probability is not a probability of infinite slip, but the probability at which the mobile 
segments have moved over a finite distance $D$, at which a final event occurs: in this case, the probability would be the solution of the equation:

$$
\frac{1-\left(\mathrm{cF}_{\mathrm{c}}\right)^{\mathrm{r}}}{1-\mathrm{cF}_{\mathrm{c}}}=\mathrm{a}_{M}
$$

reducing to:

$$
\frac{a}{1-c F_{c}}=a_{M}
$$

If the finite number $r$ of elementary cells swept over is large enough to neglect the $\left(c F_{c}\right)^{r}$ term.

In such a description, the function (22) can be considered as a particular distribution function, and, as shown on Fig. 6 for $c=2.5$, very similar to the previously reported ones in the $\{0,1\} z^{g}$ domain (F1g.3 or 5), when normalized and still choosing the critical value for $z^{g}=1$.
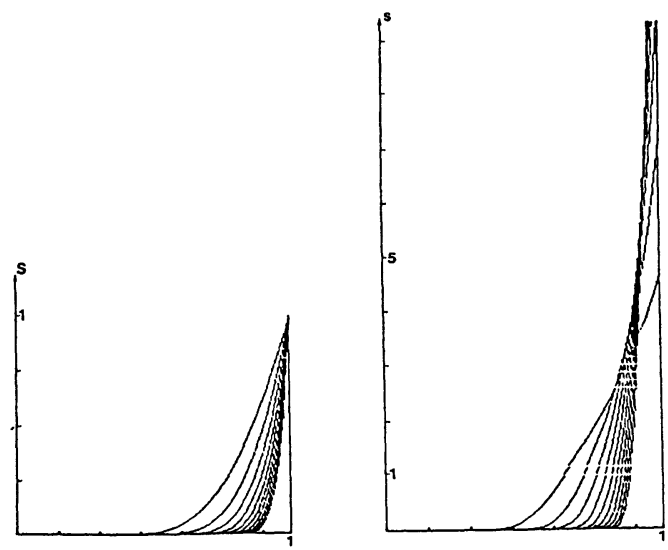

Fig. 6. The function (22) for finite $\operatorname{silp}$ at $z^{g}=1$

This does not allow to conclude whether or not the general solution of (50) always correspond with non zero $\dot{\mathrm{i}}^{l}$ values, say an asymptotical threshold for the steady state plasticity. However, if the solution always corresponds with such an asymptotical steady state, it does not matter whether the AS value is infinite or not at a critical $z^{\ell}$ value, here chosen equal to unity for simplicity.

The next part 1llustrates how the flow solution can be obtained from the present representation, when the yield surface is only an asymptotical limit.

\section{III.3. Illustrative exemples}

The Fig. 7 and 8 illustrate the flow solution obtained from the equations (52) and (43) for an imaginary material with respectively one single slip system and two slip systems, in the case of an asymptotical threshold for steady state, and using a power law for both $S\left(S=\left(z^{8}\right)^{m}\right)$ and $D\left(D=\left(z^{8}\right)^{p}\right)$ :

- In the former exemple, assuming a single slip system, (Fig. 7) there is no roiation and $\dot{\sigma}$ being the load parameter one has, from (47) $C=\dot{\tau}=R \dot{\sigma}$ and $\Delta \mathrm{h}=0$, what gives, using (52):

$$
\dot{z}=\frac{\left(C-z T L, \theta^{S(z))}\right.}{\tau_{c}+z T s^{\prime}(z)}
$$

If from (57), $\dot{z}$ generally decreases with the load increase, it may or may not reach the zero value according to $\mathrm{L}, \theta$ which is the only unprescribed parameter. Setting:

$$
\mathrm{L}, \theta=\mathrm{C} / \mathrm{T}
$$

assignes a zero $\dot{z}$ value for $z=1$ what ensures the asymptotical behaviour as reported on Fig. 7.

This $\tau$ versus $\gamma$ curve is typical of the single crystal single slip straining, with the elastic plastic transition domain. The critical shear stress, equal to $\tau_{0}$ for the unloaded materlal, Increases with the load so as to always remain higher than the applied resolved shear stress. This initial domain sharpens when the power is increased in $S$ and $D$, to disappear when $D$ is a Dirac function.

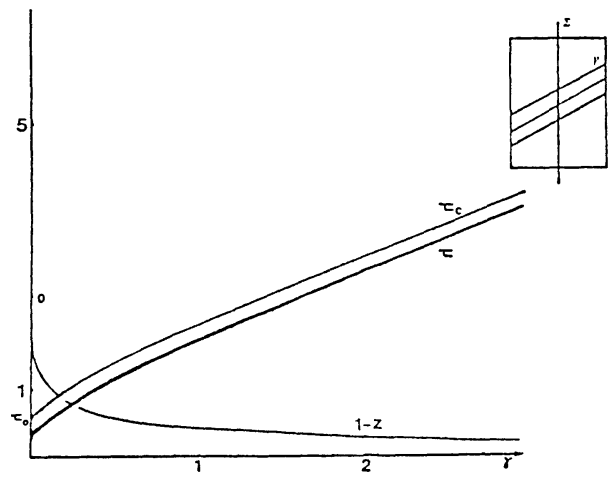

Fig. 7 Simulated $\tau-\gamma$ curve for asymptotical steady state, one slip system, no rotation

- In the second exemple, we consider two slip systems, $\mathrm{n}^{\circ} 1$ and $\mathrm{n}^{\circ} 2$, and an initially predominant number one, and we account for a rotation like effect which increases the applied resolved shear stress rate on the latent $\left(n^{\circ} 2\right)$ system during primary slip (we here simply assume the rotation 
rate of the tensile axis towards the primary slip diection proportionnal to $\dot{\gamma}^{1}-\dot{\gamma}^{2}$ ). Under the assumption of an asymptotical threshold, one has to solve, from the right hand side of $(52 b)$, the following equalities:

$$
\begin{aligned}
& C^{1}+\left(b \Delta h^{11}-T^{11}\right) L_{, \theta}^{1}+\left(b \Delta h^{12}-T^{12}\right) L_{, \theta}^{2}=0 \\
& C^{2}+\left(b \Delta h^{21}-T^{21}\right) L_{, \theta}^{1}+\left(b \Delta h^{22}-T^{22}\right) L_{, \theta}^{2}=0
\end{aligned}
$$

what determines the unprescribed $L_{, \theta}^{\ell}$ parameters according to the specified assumption - which are equivalent to (58) for a single slip system - and then the $\dot{z}^{l}$ terms are calculated. The Fig. 8 reports the $\tau 1$ versus $\gamma 1$ curve now including a stage two like domain, still with an asymptotical approach of the critical shear stress. The corresponding curve obtained without rotation, is also reported for comparison.

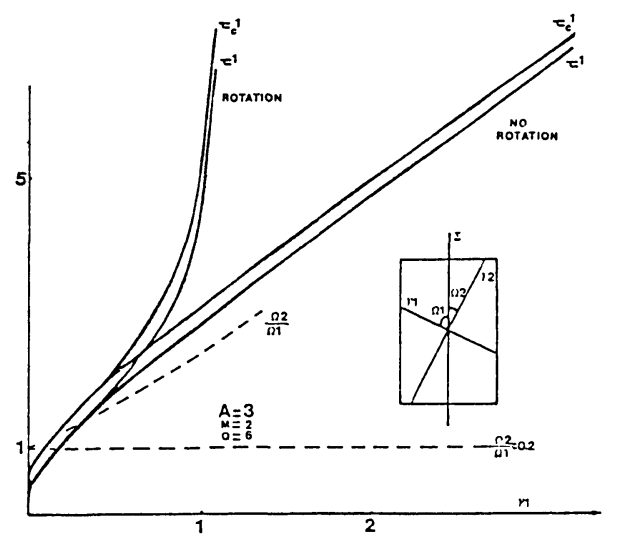

Fig. 8 Simulated $\tau^{1}-\gamma^{1}$ curve for asymptotical steady state, two slip systems, a single active one $\left(n^{\circ} 1\right)$, with and without rotation effect
CONCLUSION.

The present approach, based on the introduction of dislocation segment distributions in the primary dislocation density and in the slip expressions of each slip system, provides a general form of the plastic flow solution which allows to discuss the major usual phenomenological descriptions, for both flow and work hardening, as particular approximations. It appears that :

- the rate independent plasticity can here be interpreted as a dislocation segment displacement law of a Dirac form.

- the strain hardening law appears spectfically related with the extremal case of a Dirac function too for the dislocation segment distribution, corresponding with the Schmid law flow criterion and having to be treated as the singular limit of the general approach here derived.

- when used with the rate independent Schmid flow criterion, the previously introduced generalized hardening law is conventent for any other dislocation segment distribution function, and accounts for the presteady state hardening features, say essentially the hardening contribution of the Inactive systems during plastic flow.

- when neither the dislocation segment distribution nor the dislocation segment displacement law are Dirac functions, the solution provided by the present generalized plastic flow criterion, can a priori elther correspond with a true yield point or with an asymptotical steady state threshold, according to the precise expressions chosen for the slip and primary dislocation density functions. This latter case corresponds with a rate dependent plastic flow description of the known type from the 1iterature. The analysis of this generalized flow criterion is still in progress so far.

\section{REFERENCES}

Asaro R.J., Needleman A, (1985) Acta Metall 33 p 923

Kocks U. F., (1967) Can J Phys 45 p 737

Franciosi P., (1984) Doctoral thesis Univ. Paris Nord, France

Franciosi P., (1985) Acta Metall 33 p 1601

Francios1 P., (1985) Toronto ASM falls meeting p 43

Franciosi P., (1987) Proc 8th RISO symposium, Rotskilde p 83

Hutchinson J.W., (1976) Proc R Soc London 348 p 101 Kocks U. F., (1966) Phil Mag 13 p 541
Lavrentev F. F., (1976) ICSMA 4 Vl p 85

Morzik H., Rompagel D. Jager F., (1987) Proc 8th RISO symposium, Rotskilde p 433

Orowan E., (1940) Proc Phys Soc 52 p 8

Schmid E., Siebel G., (1931) Z Electrochem 37 p 447

Taylor G.I., (1934) Proc R Soc A 145 p 362

Z^rka J., (1972) J Mech Phys Solids 20 p 179 